\title{
Effect of Sniffing on the Temporal Structure of Mitral/Tufted Cell Output from the Olfactory Bulb
}

\author{
Ryan M. Carey ${ }^{1}$ and Matt Wachowiak ${ }^{1,2}$ \\ ${ }^{1}$ Department of Biomedical Engineering, Boston University, Boston, Massachusetts 02215, and ${ }^{2}$ Department of Physiology and Brain Institute, University of \\ Utah, Salt Lake City, Utah 84108
}

\begin{abstract}
Neural activity underlying odor representations in the mammalian olfactory system is strongly patterned by respiratory behavior. These dynamics are central to many models of olfactory information processing. We have found previously that sensory inputs to the olfactory bulb change both their magnitude and temporal structure as a function of sniff frequency. Here, we ask how sniff frequency affects responses of mitral/tufted (MT) cells-the principal olfactory bulb output neurons. We recorded from MT cells in anesthetized rats while reproducing sniffs recorded previously from awake animals and varying sniff frequency. The dynamics of a sniff-evoked response were consistent from sniff to sniff but varied across cells. Compared to the dynamics of receptor neuron activation by the same sniffs, the MT response was shorter and faster, reflecting a temporal sharpening of sensory inputs. Increasing sniff frequency led to moderate attenuation of MT response magnitude and significant changes in the temporal structure of the sniff-evoked MT cell response. Most MT cells responded with a shorter duration and shorter rise-time spike burst as sniff frequency increased, reflecting increased temporal sharpening of inputs by the olfactory bulb. These temporal changes were necessary and sufficient to maintain respiratory modulation in the MT cell population across the range of sniff frequencies expressed during behavior. These results suggest that the input-output relationship in the olfactory bulb varies dynamically as a function of sniff frequency and that one function of the postsynaptic network is to maintain robust temporal encoding of odor information across different odor sampling strategies.
\end{abstract}

\section{Introduction}

Active sensing allows an animal to control the interaction between a stimulus and the sensory neurons detecting it. Whisking, licking, and saccadic eye movements are classical examples of active sensing behaviors; in olfaction in terrestrial vertebrates, odor sampling is controlled by the inhalation of air into the nose, which can occur in the course of baseline respiration or during active sensing (i.e., "sniffing"). The dependence of sensory neuron activation on respiration has profound consequences for olfactory system function.

First, respiration imposes a temporal structure on the activation patterns of olfactory receptor neurons (ORNs), with ORNs typically firing in 100-200 ms bursts of activity following inhalation (Spors et al., 2006; Carey et al., 2009). ORN input dynamics in turn appear to shape the temporal response patterns of mitral/ tufted (MT) cells, the main output neurons of the olfactory bulb (OB) (Sobel and Tank, 1993). Temporal patterns of inhalationdriven ORN and MT cell spike bursts may by themselves robustly encode information about odorant identity and intensity (Cha-

Received April 11, 2011; revised May 16, 2011; accepted June 3, 2011.

Author contributions: R.M.C. and M.W. designed research; R.M.C. performed research; R.M.C. analyzed data; R.M.C. and M.W. wrote the paper.

This work was supported by NIH Grants R01 DC06441 (M.W.) and F31 DC010312 (R.M.C.). We thank lan Davison, Tristan Cenier, and Yusuke Tsuno for assistance with the extracellular recording protocol, and Don Katz and Markus Rothermel for discussion and comments on this manuscript.

The authors declare no competing financial interests.

Correspondence should be addressed to Matt Wachowiak, Department of Physiology, Brain Institute, University of Utah, Salt Lake City, UT 84103. E-mail: matt.wachowiak@utah.edu.

DOI:10.1523/JNEUROSCI.1805-11.2011

Copyright $\odot 2011$ the authors $\quad 0270-6474 / 11 / 3110615-12 \$ 15.00 / 0$ put, 1986; Hopfield, 1995; Margrie and Schaefer, 2003; Schaefer et al., 2006; Cury and Uchida, 2010; Junek et al. 2010).

Second, sniffing alters the strength and temporal organization of ORN activation patterns. Sniffing involves precise but complex control of the frequency and magnitude of inhalation during behavior (Welker, 1964; Youngentob et al., 1987; Kepecs et al., 2007; Wesson et al., 2008b). The most prominent form of active sniffing involves repeated inhalation at high frequencies $(6-10$ $\mathrm{Hz}$ ); in rodents this behavior is expressed during active exploration, investigation of novel stimuli and odor learning (Welker, 1964; Macrides et al., 1982; Wesson et al., 2008a,b). Sustained high-frequency sniffing alters patterns of ORN activation by attenuating the magnitude of sniff-evoked responses and reducing the temporal coherence between sniffing and ORN spiking (Verhagen et al., 2007; Carey et al., 2009).

Changes in sniffing behavior during active sensing may also alter patterns of postsynaptic activity in the OB and beyond. Indeed, changes in the frequency and temporal structure of ORN input are predicted to lead to qualitative changes in the way that the $\mathrm{OB}$ processes odor information during active sampling (Schoppa and Westbrook, 2001; Balu et al., 2004; Hayar et al., 2004a; Wachowiak and Shipley, 2006). However only a few studies have recorded MT cell responses during sniffing in the highfrequency $(6-10 \mathrm{~Hz})$ range (Bhalla and Bower, 1997; Kay and Laurent, 1999; Bathellier et al., 2008; Cury and Uchida, 2010), and none have provided a detailed characterization of how (or whether) the transition from passive respiration to sustained high-frequency sniffing systematically alters the response properties of $\mathrm{OB}$ neurons. 
Here, we recorded from MT cells in anesthetized rats while using a "sniff playback" device to reproduce sniffing behavior recorded previously from awake animals (Cheung et al., 2009). This approach allowed us to investigate how sniff frequency affects MT cell responses under naturalistic sampling conditions. We found that increasing sniff frequency altered the shape of the inhalation-evoked MT cell response transient and did so in a way that enabled a population of MT cells to maintain strong respiratory patterning across the range of sniff frequencies expressed during behavior.

\section{Materials and Methods}

Sniff playback. Female Long Evans rats (9-15 weeks old; mean body mass, $243 \mathrm{~g})$ were anesthetized with urethane $(1.7 \mathrm{~g} / \mathrm{kg})$ or isoflurane (1-3\%) and kept on a heating pad for the duration of the experiment. The sniff playback technique used here, including sources and part numbers, is described in detail by Cheung et al. (2009). Briefly, a tracheotomy was performed, and a breathing tube was inserted toward the lungs. A second tube was inserted via the trachea into the nasopharynx and secured with surgical thread and VetBond. The sniff tube was connected through a short length of polyethylene tubing to a glass syringe, the piston of which was coupled to a computer-controlled linear solenoid actuator.

Playback command signals for the solenoid were derived from intranasal pressure signals recorded previously from awake head-fixed rats (Verhagen et al., 2007). We used two different playback traces; the first replicated a $10 \mathrm{~s}$ recording of sniffing in an awake rat that consisted of both low- and high-frequency sniffing (see Fig. $1 A$ ), and the second trace consisted of bouts of sniffs repeated at each of five frequencies from 1-5 $\mathrm{Hz}$ with a pause of $2 \mathrm{~s}$ in between each bout (see Fig. $1 B$ ). Because the waveform of individual sniffs varies with frequency (high-frequency sniffs tend to be shorter), for each frequency the sniff waveform in a bout was taken from the average of many $(>100)$ sniffs recorded at that frequency. The average sniff for a given frequency range was generated by recording all sniffs from long (30-45 min) sessions of sniffing behavior (Carey et al., 2009) and binning each sniff by its preceding intersniff interval (ISI). Sniffs in each bin (100 ms wide; bin centers were 1000, 500, 400,300 , and $200 \mathrm{~ms}$, corresponding to sniffing at $1,2,2.5,3.3$, and $5 \mathrm{~Hz}$, respectively) were averaged and the resulting average sniff for each frequency bin used to synthesize the playback traces. For each playback trace, the sniff waveform used for any given sniff was the average sniff calculated for that sniff's ISI. This approach allowed us to examine responses to a particular sequence of sniffs and to control for potential effects of changes in sniff waveform. The gain of each of the command traces was adjusted during playback such that the working range of the syringe was $\sim 1.5 \mathrm{ml}$, an appropriate tidal volume for an $\sim 250 \mathrm{~g}$ rat (Walker et al., 1997). The pressure transients delivered to the nose were recorded with a pressure sensor (24PCAFA6G; Honeywell) to monitor the timing and reliable reproduction of the sniff playback signals. The animal's intrinsic respiration was monitored by placing a photodiode under the chest; each breath modulated light access to the photodiode.

Odorant presentation. Odorants were presented using a custom flowdilution olfactometer controlled by a computer (Verhagen et al., 2007). Pure liquid odorants (Sigma-Aldrich) were diluted in mineral oil such that the saturated vapor contained $\sim 200 \mathrm{ppm}$ of odorant; this vapor (in pure nitrogen) was further diluted by clean air in a mixing manifold to yield an odor stream containing $\sim 2-10 \mathrm{ppm}$ final odorant concentration. Odorant was presented for the entire duration of the sniff playback trace. Stability of odorant concentration for the duration of the presentation was confirmed using a portable photoionization detector (miniRAE2000; RAE Systems).

Optical imaging. ORNs were loaded with calcium-sensitive dye, and optical signals were imaged from the dorsal $\mathrm{OB}$ as described previously (Wachowiak and Cohen, 2001; Verhagen et al., 2007; Carey et al., 2009). Briefly, rats were anesthetized with ketamine/xylazine and ORNs in both nares loaded with calcium-sensitive dye (Oregon Green BAPTA-1 dextran; $10 \mathrm{kDa}$ molecular weight; Invitrogen). Imaging was performed through an optical window $(\sim 3 \mathrm{~mm}$ anteroposterior by $1.5 \mathrm{~mm}$ medio- lateral) made by thinning the bone over one OB. Optical signals were collected using a $4 \times 0.28$ NA objective, an Olympus epifluorescence illumination turret (BX51), a 150W Xenon arc lamp (Opti-Quip), and appropriate filter sets (Verhagen et al., 2007). Images were acquired using a $256 \times 256$ pixel CCD and digitized at $25 \mathrm{~Hz}$ along with the sniff playback signals using an integrated hardware/software package (NeuroCCD SM-256 and NeuroPlex, RedShirtImaging).

Analysis of imaging data was performed mostly as described previously (Wachowiak and Cohen, 2001; Verhagen et al., 2007; Carey et al., 2009). Regions of interest representing distinct glomeruli were selected manually based on the discrete appearance of signal foci of appropriate size $(75-150 \mu \mathrm{m})$. For each glomerulus, we automatically detected responses to each sniff using a custom curve-fitting algorithm implemented in MATLAB (Carey et al., 2009). All glomerulus-odor pairs used for analysis were tested at subsaturating odorant concentrations (i.e., we confirmed that a higher concentration elicited stronger response amplitudes for each glomerulus). Raw calcium signals are shown for display in Figure 1.

For analysis of ORN input dynamics, we estimated temporal patterns of action potential firing across the ORN inputs to a glomerulus using temporal deconvolution as described by Yaksi and Friedrich (2006) and Verhagen et al. (2007). The average ORN response generated by a sniff was calculated from the deconvolved calcium signal by averaging across all sniffs repeated at a given frequency, aligned to the time of inhalation onset (see Fig. 1G); each "sniff-triggered" average response was then fit with a double sigmoid curve to measure temporal dynamics parameters (Carey et al., 2009) (see also Analysis, below). Curve fitting was only performed for responses that were strongly modulated by sniffing [e.g., those with a "sniff modulation index" (SMI; see Analysis, below, for definition) of at least 0.5].

Coherence between ORN inputs and sniffing (see Fig. $1 F$ ) was calculated from the deconvolved ORN signal as done previously (Carey et al., 2009): First, to ensure the measurement was limited to the effect of sniff frequency (rather than sniff shape or amplitude), we replaced each sniff with an averaged sniff waveform. The coherence between this modified sniff waveform and the deconvolved ORN input waveform was then calculated for each glomerulus for the duration of each bout between the second and the last sniffs.

Extracellular recordings. Extracellular recordings from OB units were performed in separate animals from the imaging experiments. Following the double-tracheotomy surgery (described above), a small $(\sim 1 \times 1$ $\mathrm{mm}$ ) craniotomy was performed over one $\mathrm{OB}$, and the dura was removed. Unit recordings were obtained using a single-channel tungsten microelectrode (2.0 M $\Omega$ at $1 \mathrm{kHz}$; MicroProbes WE3PT12.0F3) amplified with a BMA-931 amplifier and Super-Z headstage (CWE), bandpass filtered from $1 \mathrm{~Hz}$ to $10 \mathrm{kHz}$, and digitized at $20 \mathrm{kHz}$ using a Micro1401 MkII DAQ board and Spike2 software (Cambridge Electronic Design). Additional high-pass filtering was done in Spike2 at a cutoff frequency of $200 \mathrm{~Hz}$. Custom scripts in Spike2 were also used to control odorant presentation.

Recordings from presumptive MT cells were obtained by slowly lowering the recording electrode to the appropriate depth for the mitral cell layer of the dorsal or ventral OB; electrode depth was monitored with a piezoelectric micromanipulator with digital readout (Sutter Instrument MP-225). The location of the mitral cell layer was determined using a stereotaxic atlas and, in some experiments, confirmed by locating the inversion point of local field potentials evoked by lateral olfactory tract stimulation. During this phase, sniff playback was used to generate ongoing "resting" sniffs at $1 \mathrm{~Hz}$ in the absence of odorant. We selected only units that appeared well isolated, in the vicinity of the mitral cell layer, and which showed clear spiking activity in the absence of odorant. Once a target unit was chosen, odorants effective at eliciting excitatory responses at low to moderate concentrations were identified using a rapid screening procedure (Davison and Katz, 2007). Premade mixtures of six to seven odorants each were presented successively, after which the components from the most effective mixture were tested. The lowest effective concentration of the most effective odorant was then used for the duration of the recording for that target cell (typical concentration was 3-5 $\mathrm{ppm}$; concentrations used ranged from 1-10 ppm). Odorants (and num- 
ber of cells) used in the final dataset were as follows: 2-butanone (one), 2-heptanone (three), 2-hexanone (two), 2-methyl-2-butenal (five), 2-methyl butyraldehyde (one), 2-octanone (three), acetophenone (one), benzaldehyde (five), butanal (one), heptaldehyde (four), heptanal (two), hexanal (two), isoamyl acetate (one), isovaleraldehyde (two), octanal (two), and valeraldehyde (two). The typical recording sequence for a target cell consisted of at least 15 trials, during each of which the odorant was presented continuously during the sniff playback command trace. In some cells, we varied this basic experiment by interleaving trials using different sniff playback command traces or with different odorant concentrations. In all cases, the intertrial interval was at least $60 \mathrm{~s}$.

Analysis. Though we limited targeted cells to those that were well isolated during baseline sniffing, odorant presentation tended to elicit excitatory responses in additional units, necessitating offline spike sorting. Spike sorting for each recording was performed in Spike2 and was based on spike shape and amplitude and confirmed by the distribution of interspike intervals. Only cells with spikes that were clearly separable by this criteria were included in the dataset. After spike sorting, all data other than spike times were discarded; subsequent analyses were performed using MATLAB (Mathworks). For each unit, we constructed snifftriggered perisniff time histograms (PSTHs) for sniff-evoked responses at each frequency of sniffing by compiling spike counts in $10 \mathrm{~ms}$ time bins for all sniffs repeated at a given frequency (see Fig. $2 B$, blue trace). Thus, all PSTHs reflect average activity evoked by many (typically $>50$ ) sniffs. For most analyses (except where stated), we used only the third and subsequent sniffs in each bout, as the first sniff had no definable frequency and the second was often difficult to distinguish from the first response, especially at the higher sniff frequencies. Spike counts were divided by the number of sniffs and the bin width and expressed as spikes per second in all figures and subsequent analyses.

The dynamics of sniff-evoked responses were measured by fitting double-sigmoid curves to the PSTHs (for this case generated with $5 \mathrm{~ms}$ bins) for each unit at each sniff frequency (see Fig. 2C) (Carey et al., 2009). Curve fitting was used to measure peak firing rate, rise time (time from $10 \%$ to $90 \%$ of peak firing rate), and duration (response width at $50 \%$ of peak firing rate). For summary data involving durations and rise times, units with multiple peaks $(N=6)$ were omitted. For Figure 5, peak firing rate and total spike count were normalized for each MT cell by dividing by the value of that parameter for the $1 \mathrm{~Hz}$ response. We defined response onset time as the time from each sniff to the first spike for which the instantaneous firing rate (measured from each spike to the next) exceeded the spontaneous firing rate by one SD. This spontaneous firing rate was calculated by averaging the firing rate in the first $200 \mathrm{~ms}$ after each sniff without odorant presentation. Response latencies reported are the mean onset time across all sniffs. The SD of these onset times reflected the precision of spike timing. Response dynamics and response variance were also measured from single trials by calculating the instantaneous firing rate at each spike time and calculating the mean and SD of the resultant waveform across sniffs. As expected, the mean firing rate (see Fig. $2 B$, black trace) approximated a smoothed version of the PSTH.

To test whether sniff frequency alters MT response patterns, we compared the PSTHs for sniffs at different frequencies within the same MT cell using a two-sample Kolmogorov-Smirnov test to compare the distributions of sniff-triggered spike times between 1 and $5 \mathrm{~Hz}$ sniff responses. For comparing response dynamics across frequency (see Fig. $8 B, C)$, response latencies were normalized for each cell by subtracting the latency of the $1 \mathrm{~Hz}$ response and durations were normalized by dividing by the duration of the $1 \mathrm{~Hz}$ response. Latencies with respect to sniff phase were calculated by dividing the latencies by the inter-sniff interval and then subtracting the $1 \mathrm{~Hz}$ response latency. Durations with respect to sniff phase were calculated by dividing each duration by the inter-sniff interval.

A sniff modulation index (SMI) was used to measure the degree to which MT cell activity was modulated by the sniff cycle relative to peak firing rate. To calculate this index, first the PSTH (bin size, $10 \mathrm{~ms}$ ) was slightly smoothed by convolving it with a Gaussian (SD, 1 bin). The SMI was then defined as $\left(\mathrm{PSTH}_{\max }-\mathrm{PSTH}_{\min }\right) / \mathrm{PSTH}_{\max }$, where $\mathrm{PSTH}_{\max }$ and $\mathrm{PSTH}_{\text {min }}$ equal the peak and subsequent trough, respectively, of the smoothed PSTH during a bout of repeated sniffs. Thus, an SMI of 1 indicates that, for a given MT cell and sniff frequency, the PSTH minimum was 0 (i.e., there was no MT cell activity in at least one $10 \mathrm{~ms}$ bin per sniff cycle), and an SMI of 0 indicates that the PSTH was completely flat over the entire cycle from one sniff to the next (i.e., there was no modulation by sniffing). Because the PSTH is the average response across many ( $>50)$ sniffs, chance variability among bins is expected to be smoothed by averaging, and the contribution of this variability to the SMI is low. However, to estimate the chance SMI level, a shuffled PSTH was constructed from spike rasters that were randomly shuffled in time, and the SMI was calculated for this shuffled PSTH. As expected, the shuffled SMI was low: $0.24 \pm 0.10$ for $5 \mathrm{~Hz}$ sniffing (chance SMI for individual MT cells is indicted in the figures).

Population spike histograms for the entire recorded MT cell population (see Fig. $8 A, B$ ) were generated by normalizing (to the peak) the PSTHs for each MT cell and then averaging these waveforms. Synthetic response histograms were constructed for each cell using as a source the PSTH evoked during either 1 or $5 \mathrm{~Hz}$ sniffing. Source PSTHs were normalized to their own peaks. The histogram (bin size, $5 \mathrm{~ms}$ ) was initialized to zero and then synthesized by processing each sniff time in sequence: for each sniff, the source PSTH was attenuated based on the current value of the histogram bin in which that sniff occurred. The attenuation factor was 0 (no change) if the current value was 0.2 or less; the attenuation factor scaled linearly from 0 to 1 for current values from 0.2 to 1.2 . Thus, synthesized responses saturated at $120 \%$ of the peak firing rate of the source PSTH. This nonlinear synthesis algorithm was preferable to simple convolution of the source PSTH with sniff times as it avoided unrestricted summation of responses at higher sniff frequencies. SMI values for synthetic response histograms were calculated for individual cells in the same way as for experimental data.

\section{Results}

\section{Presynaptic responses to sniffing patterns derived from behaving animals}

To systematically evaluate how variations in sampling behavior (in particular, sniff frequency) shapes MT responses, we generated naturalistic inhalations (i.e., sniffs) resembling those expressed by awake animals. To achieve this we used a sniff playback device consisting of a solenoid-driven syringe under the control of an analog command waveform. The device has been described previously (Cheung et al., 2009); however, for the purposes of this study, the key features of sniff playback are (1) the ability to reproduce the pressure transients characteristic of respiration or sniffing in the awake animal, (2) the ability to generate resultant airflow patterns mimicking those seen in the awake animal, and (3) the ability to reproduce these waveforms with high precision both within and across different animals to generate naturalistic sniff waveforms in the anesthetized animal. These features are illustrated in Figure 1, $A$ and $B$. Sniff playback allowed us to systematically explore sampling parameters and to compile data across trials and across animals without the confound of differences in odor sampling or changes in behavioral state.

Sniff playback command traces were derived from intranasal pressure measurements taken during low- or high-frequency sniffing in an awake, head-fixed rat (see Materials and Methods) (Verhagen et al., 2007). We used two different sniff playback traces. The first was derived from a $10 \mathrm{~s}$ epoch recorded from an awake, head-fixed rat, which included resting respiration and a bout of high-frequency sniffing, followed by a return to resting respiration (Fig. 1A). The second trace consisted of a series of sniff bouts at different frequencies in which the same sniff was repeated six to nine times at each of five frequencies from 1 to 5 $\mathrm{Hz}$, with a pause of $2 \mathrm{~s}$ in between each bout (Fig. $1 B$ ). This first trace allowed us to record responses to a behaviorally expressed, "natural" sequence of sniffs generated in the behaving animal, 


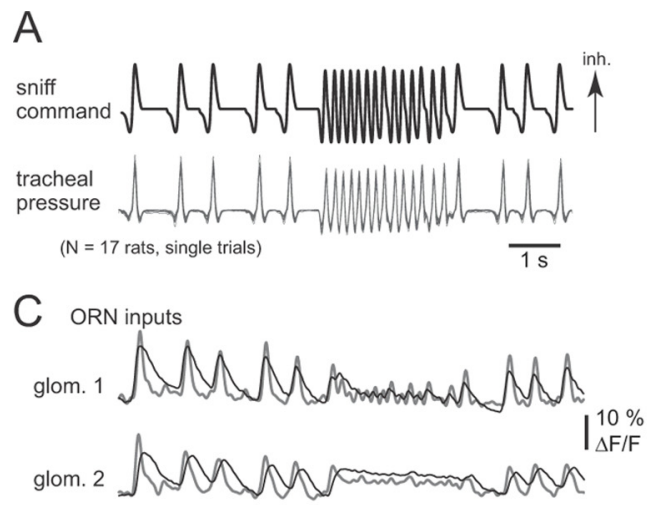

B

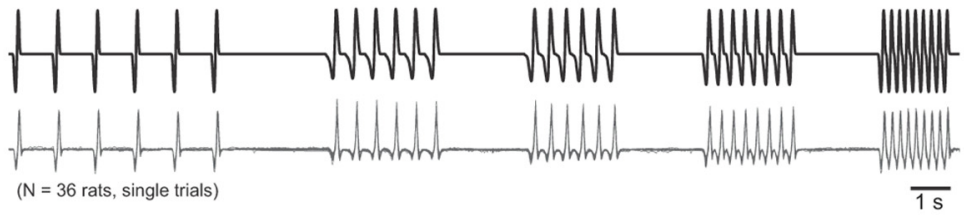

D

$\mathrm{E}$

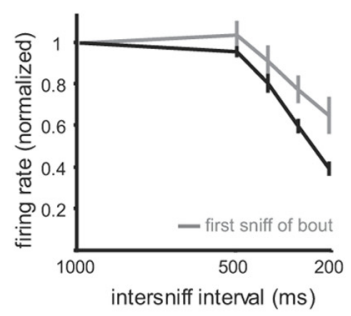

$\mathrm{F}$

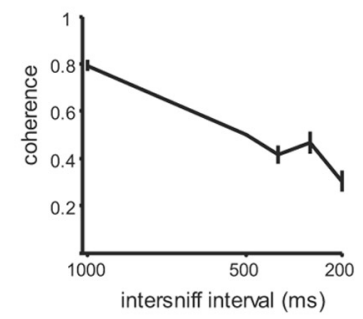

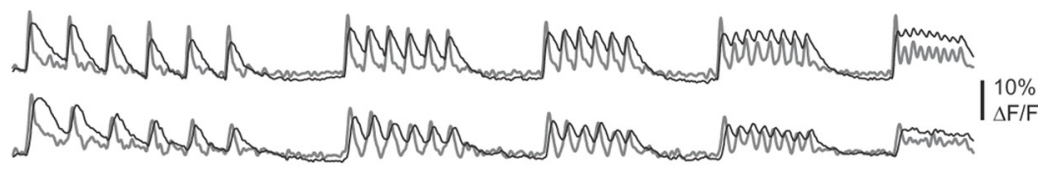

G

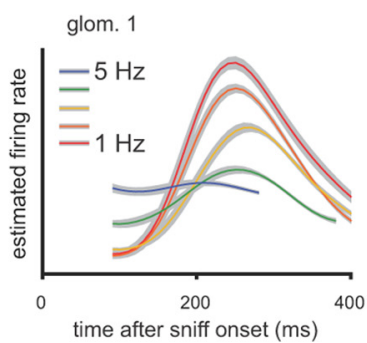

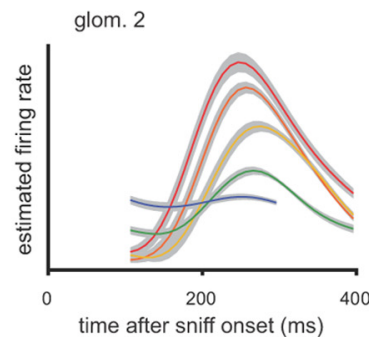

Figure 1. Sniff playback reliably drives $O R N$ input to the $O B$ in the anesthetized rat. $A$, Playback command trace reproducing sniff timing recorded from an awake, head-fixed rat (see Materials and Methods). Top trace, Playback command signal. Bottom traces, Tracheal pressure measurements from single playback trials of this command signal recorded from 17 rats. The pressure transients are highly reproducible from animal to animal. Inhalation (inh.) is up. $\boldsymbol{B}$, Synthetic playback command trace consisting of bouts of sniffing at frequencies from 1 to $5 \mathrm{~Hz}$ (top), with a $2 \mathrm{~s}$ pause between each bout. Top trace, Command signal. Bottom trace, Tracheal pressure measurements recorded during playback in 36 rats. C, D, Presynaptic calcium signals reflecting ORN input to two glomeruli (glom. 1,2) in one rat, evoked by sniff playback of the waveforms in $\boldsymbol{A}$ and $\boldsymbol{B}$, respectively. 0 dorant ( $0.2 \%$ saturated vapor ethyl butyrate) was presented for the entire duration of the playback trace. Raw optical data from imaging (average of 4 trials) are shown in black; gray traces represent estimates of firing rate changes using temporal deconvolution (see Materials and Methods). In both glomeruli, ORN input was strongly driven by each sniff during sniff playback; glomerulus 1 maintained sniff patterning during high-frequency sniff bouts, whereas glomerulus 2 did not. Both glomeruli were imaged in the same trial. $E$, Increasing sniff frequency attenuates ORN response magnitudes. Error bars represent the mean \pm SEM of the peak of the deconvolved ORN signal at each sniff frequency shown in $\boldsymbol{B}$. Responses to the third and subsequent sniffs from each bout (black) show strong attenuation; responses to the first sniff of each bout (gray) also attenuate, presumably because of differences in the waveform of the sniff generated at each frequency (see Materials and Methods). Response amplitudes (estimated firing rates) are normalized to the $1 \mathrm{~Hz}$ response for each glomerulus ( $N=33$ glomeruli from 20 rats). $\boldsymbol{F}$, Coherence of ORN inputs with sniffing also decreases with increasing sniff frequency. Error bars represent the mean $\pm S E M$ of the mean-squared coherence between the sniff pressure waveform and estimated ORN firing rate waveform for each glomerulus ( $N=33$; see Materials and Methods). Thus, sniff patterning of $0 R N$ inputs weakens at higher sniffing frequencies. $\mathbf{G}$, Sniff-triggered averages of ORN inputs for the two glomeruli shown in $(\boldsymbol{C})$ and $(\boldsymbol{D})$ at sniff frequencies ranging from 1 to $5 \mathrm{~Hz}$. Plots show the mean \pm SEM of deconvolved presynaptic calcium signal. Increasing sniff frequency decreases response amplitudes, but the shape of the response transient remains relatively invariant across frequency.

while the second trace allowed us to more systematically assess how responses vary across frequency. The interval between each sniff bout in the second trace was chosen to allow evoked responses to return to baseline; the first sniff in each bout thus had no frequency and so could serve as a control for whether sniff waveform affected responses.

We first recorded responses of ORNs to the sniff playback traces using presynaptic calcium imaging, an approach we have used previously in awake rats (Verhagen et al., 2007; Carey et al., 2009). The purpose of these experiments was to confirm that our previous observations in awake rats could be repeated using sniff playback in anesthetized rats. As expected, each sniff elicited a brief calcium signal transient reflecting a short (100-200 ms) burst of action potentials in the population of ORNs converging onto a glomerulus (Figs. 1C,D). We compared key temporal parameters of these sniff-playback-driven calcium responses (latency from sniff, rise time, and duration) with those elicited in awake rats (Carey et al., 2009) and found that these parameters closely matched. Values for the sniff playback experiments were as follows: latency, $142 \pm 58 \mathrm{~ms}$; rise time, $111 \pm 21 \mathrm{~ms}$; duration, $433 \pm 76 \mathrm{~ms}(N=33$ glomeruli). Those for awake rats were as follows: latency, $154 \pm 59 \mathrm{~ms}$; rise time, $91 \pm 40 \mathrm{~ms}$; duration, $392 \pm 149$ ms (Carey et al., 2009). At low sniff frequencies (1-2 $\mathrm{Hz}$ ), responses repeated with little decrement in amplitude for each sniff in the bout. At higher frequencies $(4-5 \mathrm{~Hz})$, the initial response (e.g., to the first sniff) was the same, but responses to subsequent sniffs were attenuated in amplitude (Figs. 1C-E). We also observed a loss of coherence between phasic ORN inputs and sniff timing as sniff frequency increased, although the degree of coherence loss differed for different odor-glomerulus pairs (Figs. $1 D, F)$. Aside from the decrease in response amplitude and coherence, the temporal structure of the sniff-evoked ORN response was relatively invariant to changes in sniff frequency (Fig. $1 G$ ). These results recapitulate those seen in awake, behaving rats (Verhagen et al., 2007; Carey et al., 2009). Thus, the sniff playback approach is capable of reproducing the key features of ORN input responses across a range of sniff frequencies.

\section{Temporal dynamics of MT cell responses driven by sniff playback}

Next we recorded from individual $\mathrm{OB}$ units during sniff playback. We focused on well-isolated units that we presumed to be MT cells based on their location and additional criteria described in Materials and Methods. We also selectively focused on units that showed excitatory responses to relatively low $(<10 \mathrm{ppm})$ concentrations of single odorants. MT cells showing purely inhibitory responses were observed but not studied further here. In total, we recorded responses from 37 cells in 20 animals, under either urethane ( $N=17$ cells $)$ or isoflurane $(N=20$ cells $)$ anesthesia. Most basic response properties did not differ between the 
A

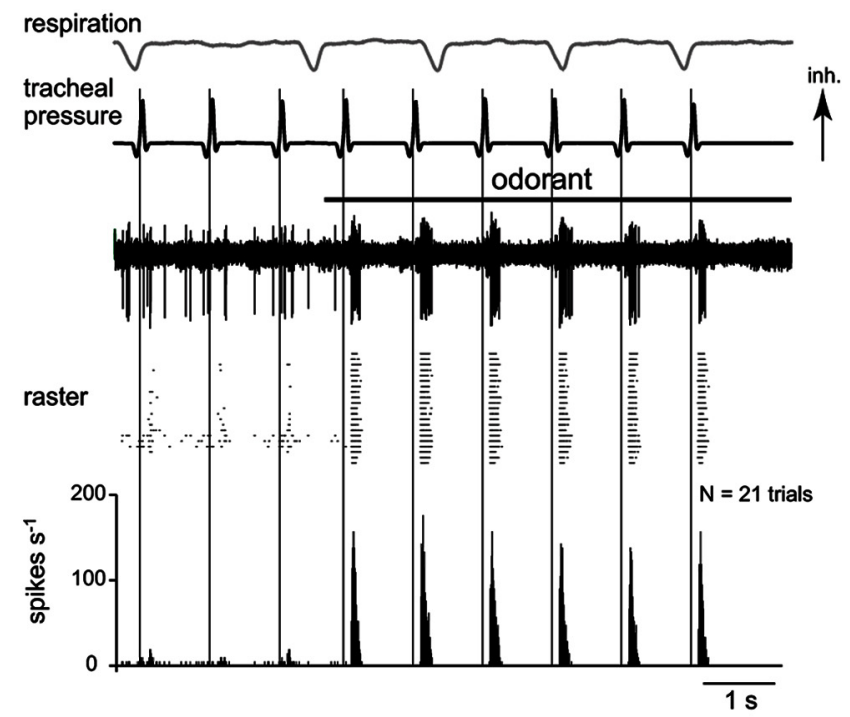

B
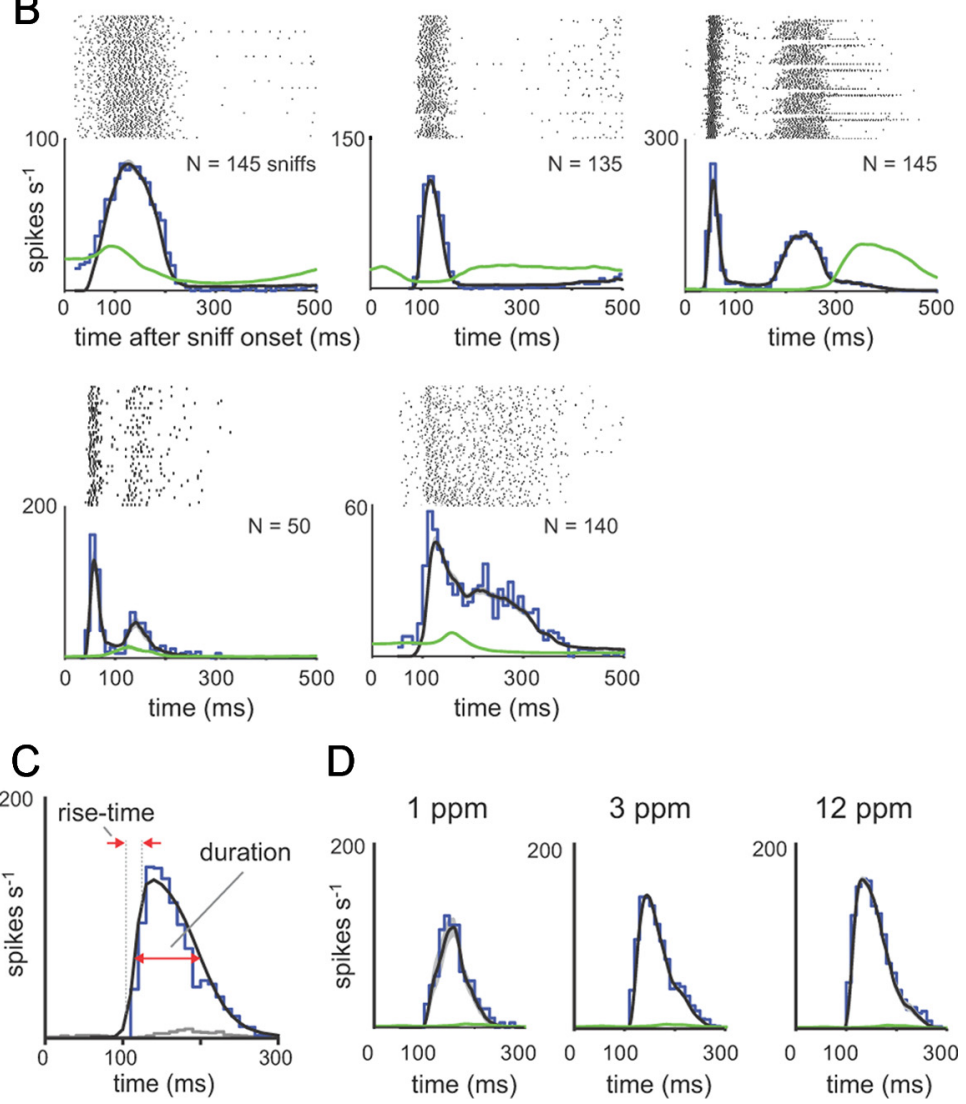

D
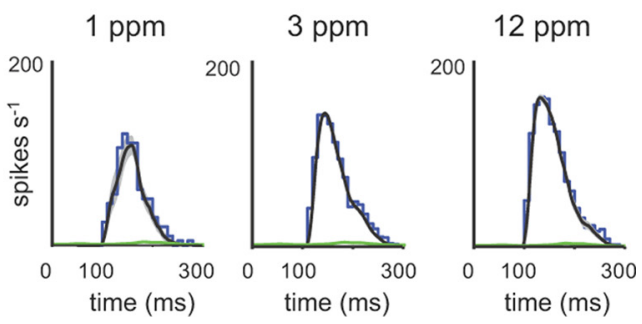

Figure 2. Sniff playback drives presumptive MT cell responses with diverse but reliable temporal dynamics. $\boldsymbol{A}$, Example recording from a presumptive MT cell during odorant presentation and sniff playback at $1 \mathrm{~Hz}$ frequency. The top three traces show, from top to bottom, intrinsic respiration measured with a photodiode, the sniff playback signal [inhalation (Inh) is up], and voltage signals from a representative playback trial in which odorant (horizontal black bar) is presented midway through the trial. Spike times from repeated trials are shown in the raster below, and the average response histogram (10 ms bins) for all trials is shown at the bottom. This unit shows weak responses to inhalation in the absence of odorant and strong excitatory transients in the presence of odorant. $\boldsymbol{B}$, PSTHs, constructed as described in Materials and Methods, from five different MT cells illustrating diverse temporal responses to odorant. For each cell, the sniff-triggered spike raster is shown along with the PSTH evoked during odorant presentation (blue plot). The mean instantaneous firing rate (black trace; see Materials and Methods) tracks the PSTH well and is reliable from trial to trial; the variance of instantaneous firing rate (measured as SEM and shown in gray overlaid on the black trace) is generally not apparent in these plots. The instantaneous firing rate measured in the absence of odorant is shown in green. Each cell shows a transient excitation, with a distinct but reliable temporal pattern. $\boldsymbol{C}$, Illustration of quantification of response dynamics using the PSTH for the MT cell shown in $\boldsymbol{A}$. Spike raster, as well as odorant-driven PSTH (blue) and nonodorant PSTH (gray) are shown. The PSTH was fitted with a double-sigmoid curve (black); this curve was used to measure peak firing rate, rise time, and duration as indicated and described in Materials and Methods. D, Example of the effect of increasing odorant concentration on MT two anesthetic regimens, except where reported below. Spontaneous spiking activity was lower and more stable using isoflurane (mean, $16 \pm 24 \mathrm{~Hz}$ spontaneous firing rate for $N=17$ cells recorded under urethane anesthesia, $14 \pm 15 \mathrm{~Hz}$ for $N=20$ cells recorded under isoflurane) (see Materials and Methods).

In most MT cells, sniff playback for an excitatory odorant elicited a brief burst of action potentials (Fig. $2 A)$. At low $(1 \mathrm{~Hz})$ sniff frequencies, this burst was repeated reliably with each successive sniff and was independent of the intrinsic respiratory rhythm. Some (but not all) MT cells showed temporal patterning of spiking in the absence of odorant; in these neurons, patterning was also linked to sniff timing and not the respiratory rhythm (data not shown). We constructed PSTHs of the response of each MT cell to a single sniff (Fig. 2B) (see Materials and Methods). In general, all cells showed a transient excitation following inhalation (we explicitly did not include cells that failed to show any excitation); however, there was considerable diversity in the specific temporal pattern of this response across cells (Fig. $2 B$ ). Sniff-triggered response patterns differed in latency to excitation, rise time of the excitatory response, and duration of excitation; in addition, some cells showed a bimodal excitation pattern with a second peak in spike frequency after the initial response transient (Fig. 2B, cells 3-5). Despite variance from cell to cell, for a given cell the basic response pattern was repeated with little variance for multiple sniffs of a given odorant.

We quantified the following key parameters of the MT cell response: peak firing rate, rise time, and duration of the average sniff-driven response (Fig. 2C), which were measured from the PSTHs, as well as mean response latency after inhalation, which was measured from individual sniffs. Details for how each parameter was quantified are given in Materials and Methods. Peak firing rates across all recorded cells varied from 38.8 to $200 \mathrm{~Hz}$ (mean, $115 \pm 35 \mathrm{~Hz}$ for $N=17$ cells recorded under urethane anesthesia, $103 \pm$ 43 for $N=20$ cells under isoflurane). In a subset of cells $(N=7)$, we presented the same odorant at multiple concentrations

$$
\leftarrow
$$

cell response dynamics. Spike rasters and PSTHs for a cell presented with odorant (heptaldehyde) at $1 \mathrm{ppm}(N=20$ sniffs), $3 \mathrm{ppm}(N=126$ sniffs), and $12 \mathrm{ppm}(N=48$ sniffs). Panel elements and colors are as in $\boldsymbol{B}$. In this cell, increasing odorant concentration increased peak firing rate and slightly shortened rise time and latency. 
A

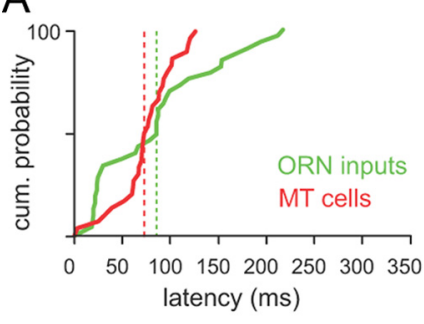

C

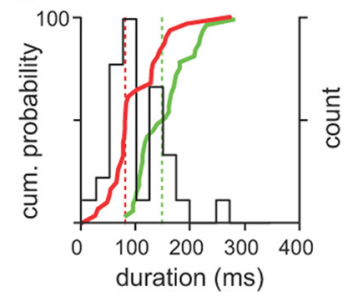

B

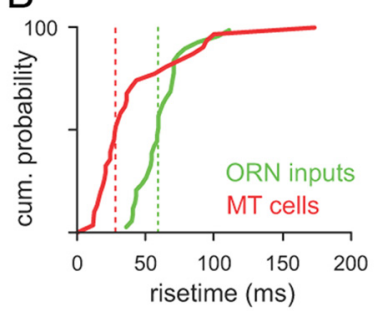

D

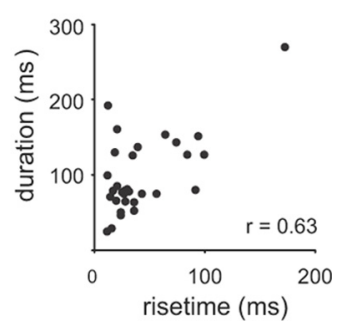

Figure 3. Temporal dynamics of MT cell responses during low-frequency sniffing. $\boldsymbol{A}$, Sniffevoked response latencies for MT cells versus ORN inputs. Plots show cumulative probability distributions of latency from inhalation to response onset for MT cell PSTHs (red) and ORN inputs (green). The median for each distribution is indicated by a vertical line. MT cell and ORN input latencies varied across a similar range ( $N=37 \mathrm{MT}$ cells; $N=33$ glomeruli for ORN inputs for $\boldsymbol{A}-\boldsymbol{C}$ ). $\boldsymbol{B}$, Response rise times for MT cells and ORN inputs; rise times of MT cell responses were shorter than those of ORN inputs. $C$, Response durations for MT cells and ORN inputs. MT cell response durations were shorter than $0 \mathrm{RN}$ input durations. The distribution of MT cell durations was bimodal, with a separation at $100 \mathrm{~ms}$ (histogram shown in black). D, MT cell response rise times and durations were highly correlated $(r=0.62)$.

(two to three concentrations per cell, ranging from twofold to 50 -fold change in concentration) (Fig. $2 D$ ). In these, peak firing rates increased with concentration (Fig. $2 D)(p<0.05$, paired $t$ test comparing lowest and highest concentrations; $N=7$ cells). Response latencies decreased slightly in most cells $(p<0.05$; paired $t$ test), while response rise times showed no significant change ( $p=0.16$; paired $t$ test). These effects of odorant intensity are similar to those observed for ORN inputs in awake rats (Carey et al., 2009).

The distributions of the temporal parameters of all MT cell responses are shown in Figure 3. Because we used the same double-sigmoid fitting method used to characterize the temporal patterning of sniff-driven ORN inputs (Carey et al., 2009), we could compare MT cell response dynamics with those of ORN inputs imaged using playback of the same sniff waveforms. In general, MT response latencies (Fig. 3A) were similar to those of imaged ORN inputs (median MT vs ORN latency, 75 vs $88 \mathrm{~ms}$ ), whereas MT response rise times (Fig. 3B) and durations (Fig. 3C) were shorter (rise times, 28 vs $60 \mathrm{~ms}$; durations, 80 vs $154 \mathrm{~ms}$ ). Notably, the distribution of PSTH durations was bimodal, with 19 cells showing durations of $<100 \mathrm{~ms}$ (mean, $66 \pm 15 \mathrm{~ms}$ ) and 12 cells showing durations of $>100 \mathrm{~ms}$ (mean, $175 \pm 44 \mathrm{~ms}$ ). Short- and longer-duration MT cells also had distinct rise times ( $30 \pm 18 \mathrm{~ms}$ for short duration, $61 \pm 48 \mathrm{~ms}$ for longer duration; $p<0.05, t$ test), and there was a high correlation between rise time and duration $(r=0.63 ; p<0.001)$ (Fig. 3D). There was no significant correlation between latency and duration $(r=0.05$; $p=0.78$ ), consistent with the idea that ORN input dynamics primarily determine MT response latency but not later aspects of the response.

\section{Precision of inhalation-driven MT cell responses}

Recent behavioral and electrophysiological studies have pointed to the importance of spike timing (in particular, first spikes) to

A
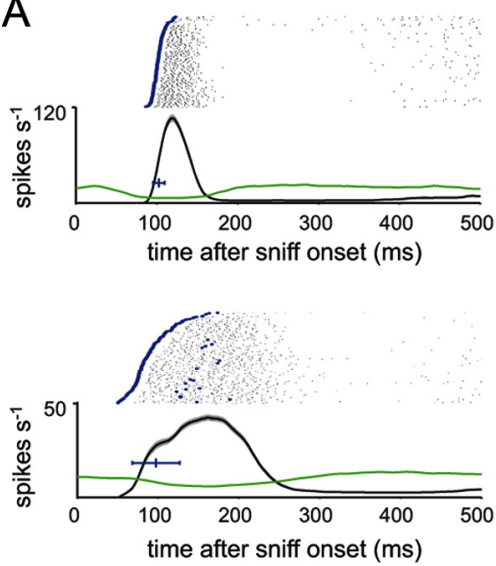

B
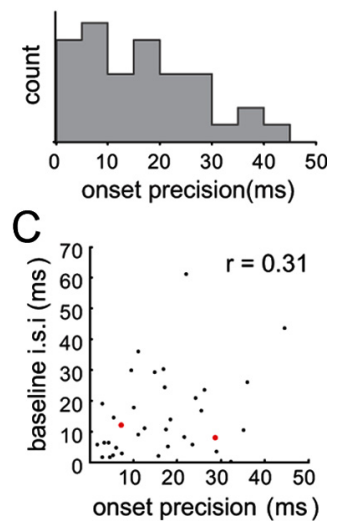

Figure 4. Precision of spike timing varies among MT cells. $\boldsymbol{A}$, Spike rasters and instantaneous firing rates (mean \pm SEM, black over gray shading), as well as preodor instantaneous firing rate (green), for two MT cells. Blue dots in spike rasters highlight the first spike after each sniff with an instantaneous firing rate that exceeds the mean preodor firing rate by one SD; the time of this first spike was the response "onset time" after each sniff. Horizontal blue bar indicates the mean \pm SD of onset time across trials. Onset precision was defined as the SD of the onset time. In this example, the mean onset times for the two cells were similar, but one cell (top) had an SD of $7 \mathrm{~ms}$, while the other had an SD of $29 \mathrm{~ms}$. $\boldsymbol{B}$, Distribution of precision times for $35 \mathrm{MT}$ cells. Precision values ranged from 2 to $44 \mathrm{~ms}$ (median, $15 \mathrm{~ms}$ ). $C$, There was no relationship between onset precision and baseline firing rate. Precision (SD of onset time) is plotted against mean baseline interspike interval for 35 MT cells. The two cells in $\boldsymbol{A}$ are highlighted in red; both had similar baseline firing rates $(\sim 10 \mathrm{~Hz})$.

odor representations and odor perception (Margrie and Schaefer, 2003; Schaefer et al., 2006; Wesson et al., 2008a; Cury and Uchida, 2010; Junek et al., 2010). We thus measured the temporal precision with which a sniff could repeatedly elicit spiking responses by measuring response onset time and its variance (SD) across repetitions of the same sniff waveform (Fig. 4A) (see Materials and Methods). Lower variance values reflect increased sniff-coupled precision of onset timing. Median precision was $15.6 \mathrm{~ms}$, and ranged from 2 to $44 \mathrm{~ms}$ (Fig. $4 \mathrm{~B})(N=35$ cells). It is possible that a MT cell's baseline firing rate or variance could affect onset precision. However, we did not find a significant correlation between onset precision and either the mean or SD of the baseline firing rate [baseline mean, $r=0.31$ (Fig. 4C); baseline $\mathrm{SD}, r=0.28]$. In addition, precision was not correlated with response amplitude $(r=-0.08)$ or latency $(r=0.17)$. Onset timing did, however, become more precise with increases in odorant concentration ( $p<0.05$, paired $t$ test comparing lowest and highest concentrations; $N=7$ cells). Thus, MT cells show different degrees of precision of spike timing across sniffs, although in general this precision is relatively high.

\section{Effects of sniff frequency on MT cell response patterns}

We next asked whether and how changes in sniff frequency alter MT responses. We analyzed MT response dynamics in the same way as described above, but now for sniffs generated at frequencies ranging from 1 to $5 \mathrm{~Hz}$. Figure 5 shows examples from two MT cells (Fig. 5A); these cells show different apparent effects of increasing sniff frequency. One cell responds with a transient burst following each inhalation at all frequencies (Fig. 5A, bottom). In contrast, a different cell responds reliably at sniffs from 1 to $3 \mathrm{~Hz}$ but begins to show attenuation in peak firing rate during sustained sniffing at 4 and $5 \mathrm{~Hz}$ (Fig. $5 A$, top). This cell also fails to return to baseline firing rate after each response, resulting in less modulation of firing by the sniff cycle. For both cells, the response to the first sniff of each bout is the same, indicating that 
A
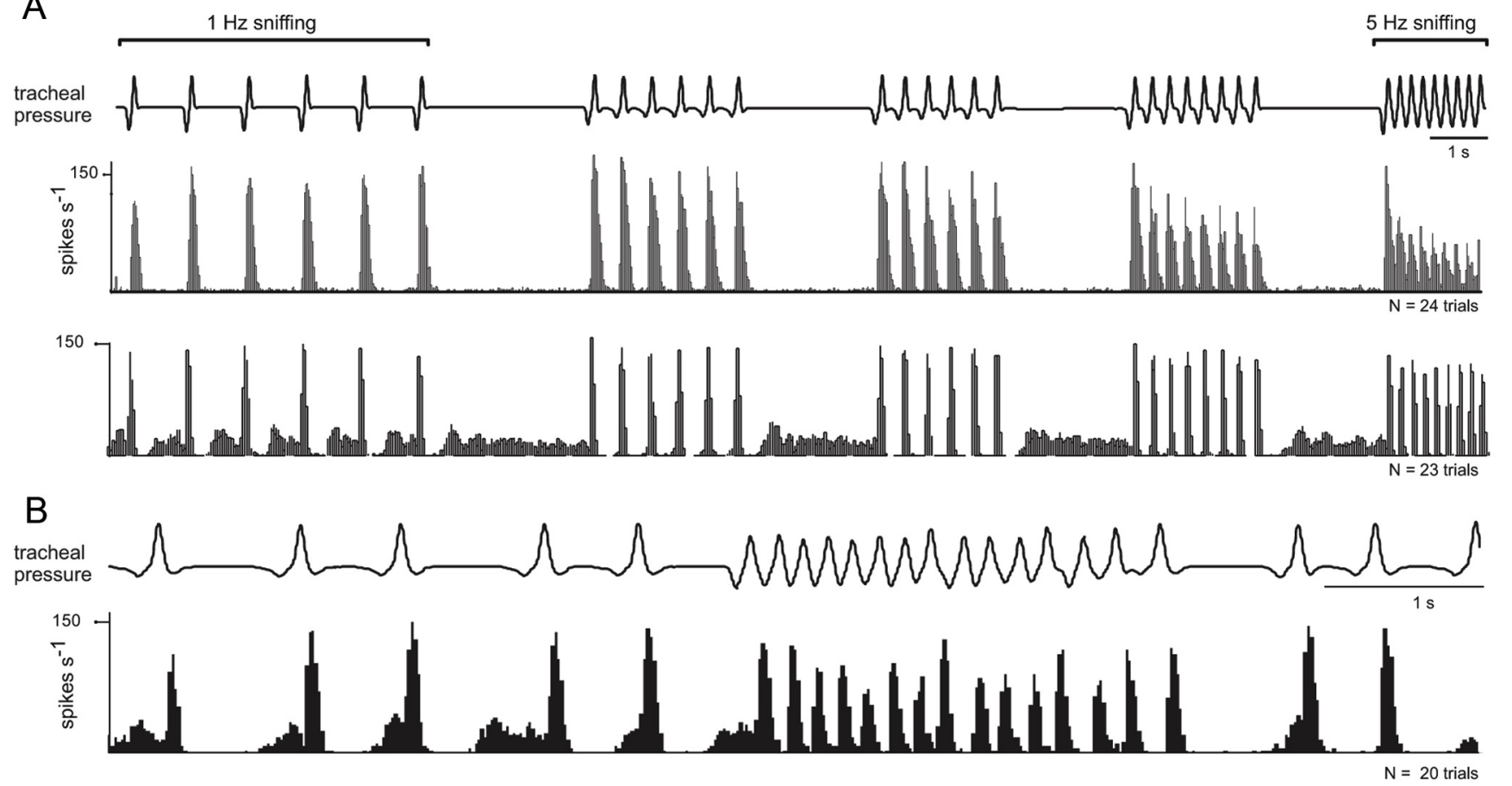

C

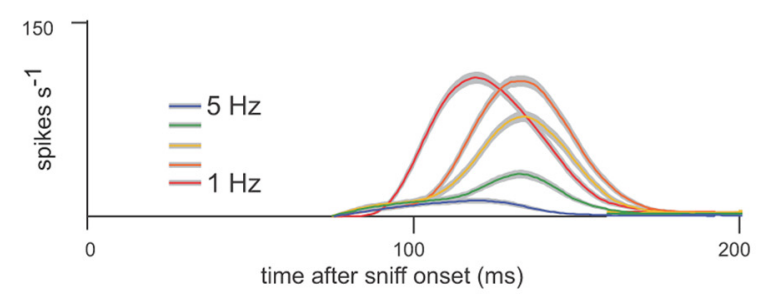

E

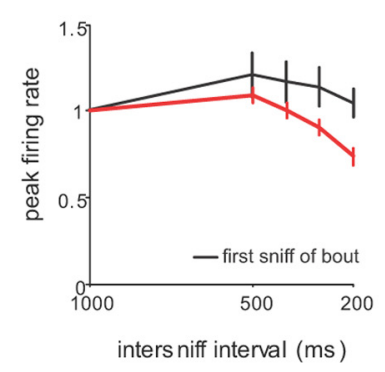

F

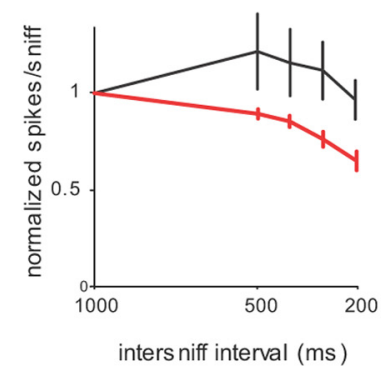

D

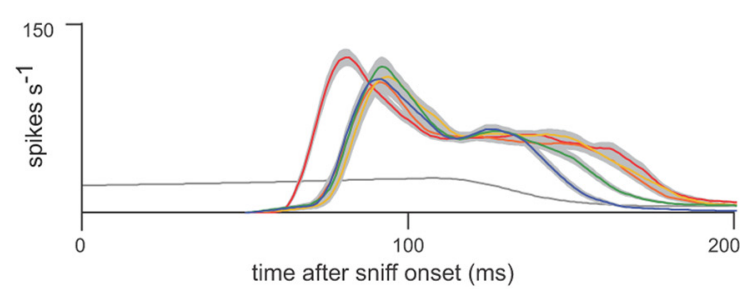

G

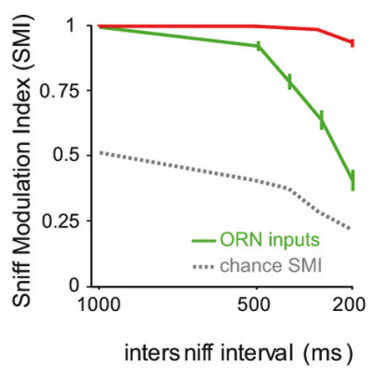

$\mathrm{H}$

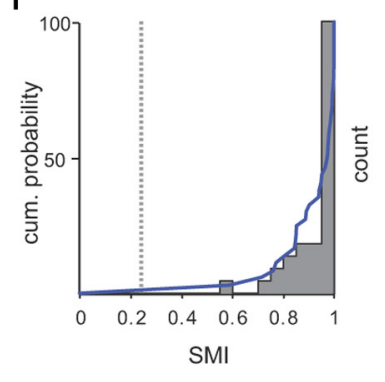

Figure 5. Sniff frequency affects MT cell response amplitudes and modulation. $A$, Responses histogram from two MT cells recorded during playback of sniff bouts at increasing frequencies from 1 to $5 \mathrm{~Hz}$. Top trace, Tracheal pressure during playback. Bottom plots, Spike histograms generated from repeated playback trials ( $N=24$ and 23 trials). 0 dorant (isoamyl acetate) was presented for the duration of the trace. In the first MT cell, responses to individual sniffs at lower frequencies were consistent throughout a bout, but as sniff frequency increased, bursts attenuated in amplitude, and responses showed less modulation bysniffing. In the second (bottom) cell, responses showed littleorno attenuation in amplitude and maintained strong modulation at high frequencies. $\boldsymbol{B}$, Spike histogram from the second cell in $\boldsymbol{A}$ during playback of the natural sniffing sequence. This cell responds faithfully to each sniff during both low-frequency sniffing and the high-frequency sniff bout. C, D, PSTHs of two different MT cells derived from sniffs at frequencies of $1-5 \mathrm{~Hz}$. Plots show the mean \pm SEM of instantaneous firing rate at each frequency. The gray line is the preodor firing rate. Only responses to the third and subsequent sniffs of each bout areincluded in this analysis. For cell $1(\boldsymbol{C}$, response amplitudes and rise times were graded by sniff frequency. For cell 2 (D), there was little attenuation with frequency, but onset latencies were longer at frequencies above $1 \mathrm{~Hz}$. $\boldsymbol{E}, \boldsymbol{F}$, Effect of snifffrequency on peak firing rate $(\boldsymbol{E})$ and number of spikes per sniff $(\boldsymbol{F})$ across all recorded MT cells $(N=37)$. Plots show the mean \pm SEM values at each frequency. Red plots show responses to the third and subsequentsniffs; black plots show responses to the first sniff in each bout. Measurements were normalized to the value at $1 \mathrm{~Hz}$ sniffing. On average, higher snifffrequencies resulted in lower peak firing rates and fewer spikes per sniff with no effect on first-sniff responses. G, SMI decreases with increased sniff frequency. See Materials and Methods for calculation of the SMI. SMI values could range from 0 to 1, with 0 indicating no modulation in firing rate by sniffing; the chance SMI (see Materials and Methods) is shown in gray. Across all cells, SMI values (red) decreased only slightly (but significantly) with sniff frequency; in contrast, SMI values for ORN inputs (green) decreased sharply as sniff frequency increased. $\boldsymbol{H}$, Distribution of SMI values for the $37 \mathrm{MT}$ cells at $5 \mathrm{~Hz}$ sniffing. Blue plot shows cumulative probability distribution; gray dotted line indicates chance SMI for $5 \mathrm{~Hz}$ sniffing. Most cells showed very little loss of modulation (SMI values near 1 ) and thus maintained patterning. The cells with the highest SMI values at $5 \mathrm{~Hz}$ were generally those with shorter durations and faster rise times (see Results).

these effects are frequency dependent and not due to the slightly different sniff waveforms at each frequency. Figure $5 B$ shows an example of the response of the lower cell from Figure $5 A$ to the natural sniff sequence; this cell responds reliably to each inhala- tion, even during the high-frequency sniff bout, which reaches sniff frequencies of $\sim 6 \mathrm{~Hz}$.

To test whether sniff frequency alters MT cell responses to the same odor, we constructed PSTHs for the average sniff-evoked 
response at each frequency (excluding the first sniff). PSTHs for two additional example cells are shown in Figure 5, $C$ and $D$. We compared PSTH shape for different frequency responses (see Materials and Methods). Every MT cell except one showed a significant change in spike distributions between and 1 and $5 \mathrm{~Hz}$ sniffing (36 of 37 cells; significant at $p<0.05$, Kolmogorov-Smirnov test). Thus, sniff frequency clearly alters MT cell response patterns.

Because ORN inputs show strong attenuation of response magnitude as sniff frequency increases (Verhagen et al., 2007; Carey et al., 2009), we predicted that MT cell responses would, on average, show a similar effect. Consistent with this prediction, we found that the peak firing rate (measured from the PSTH) decreased significantly $(p<0.001$, paired $t$ test; $N=$ 37 cells) at sniff frequencies of $3-5 \mathrm{~Hz}$ (Fig. $5 E$ ); however, the degree of the attenuation was lower than that of ORN inputs (e.g., $\sim 25$ vs $\sim 60 \%$ for $5 \mathrm{~Hz}$ sniffing). Total spike count within the $200 \mathrm{~ms}$ following inhalation also decreased (Fig. 5F) $(p<0.001$, paired $t$ test; $N=37$ cells).

Responses to the first sniff in each bout showed no change across frequencies $(p=0.77$ for effect of sniff frequency on peak firing rate; $p=0.75$ for effect of sniff frequency on spike count; ANOVA; $N=37$ cells). Increasing odorant concentration reliably increased peak firing rates, but there was no systematic effect on the degree of attenuation.

We also predicted that MT cells, like ORN inputs (Carey et al., 2009), would show less modulation in firing rate by the sniff cycle at higher sniff frequencies. Across all cells, our index of firing rate modulation by sniffing (SMI) (see Materials and Methods) decreased significantly (Fig. 5G) (paired $t$ test between 1 and $5 \mathrm{~Hz}$, $p<0.001 ; N=37$ cells) but only slightly, and less so than for ORN input signals. However, as is evident from the examples in Figure $5 A$, the ability of MT cells to maintain temporal patterning at high frequencies varied across the population. Most MT cells maintained high modulation of firing rate with the sniff cycle, with 26 of 37 cells showing almost no loss of modulation at $5 \mathrm{~Hz}$ (SMI, >0.90). However, the remaining cells did show a reduction in modulation, albeit a modest one (Fig. $5 H$ ). There was no relationship between recording depth and SMI $(r=-0.11)$, suggesting no systematic difference in sniff modulation between dorsal, lateral, or ventral MT cells, nor was there a relationship between SMI and peak sniff-evoked firing rate $(r=-0.0011)$.

In summary we found that, while the reduction in input magnitude during sustained high-frequency sniffing appears, on average, to translate into reduced MT response magnitudes, MT responses tend to maintain temporal patterning even in the face of sharply reduced temporal patterning of ORN inputs.

\section{Frequency invariance of MT response latencies}

What parameters of MT responses change with sniff frequency? We first tested the effect of sniff frequency on the timing of MT response onsets. Previous studies have proposed that odor information may be robustly represented by the relative phase of MT cell activity within the sniff cycle (Chaput, 1986; Hopfield, 1995;

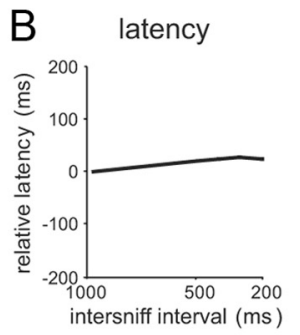

C duration
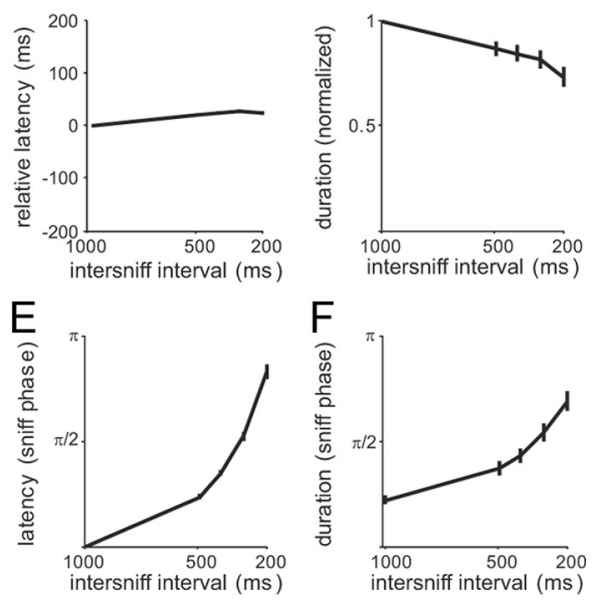

Figure 6. MT cell response patterns are relatively robust with respect to sniff onset time but not sniff phase. $\boldsymbol{A}$, Sniff-evoked (mean instantaneous spike frequency) for a cell that shows very little change in response dynamics with sniff frequency.

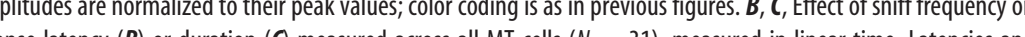
frequency on response latencies $(\boldsymbol{E})$ and duration $(\boldsymbol{F})$ analyzed with respect to sniff phase (bottom). Both latencies and durations sniff cycle with respect to sniff phase. Error bars indicate mean \pm SEM

Roux et al., 2006; Fantana et al., 2008); other studies have supported an alternative hypothesis of a temporal code based on absolute response latencies (Margrie and Schaefer, 2003; Spors et al., 2006; Junek et al. 2010). To distinguish between these two possibilities, we analyzed MT response latency and duration across frequencies, both with respect to phase of the sniff cycle and to linear time relative to inhalation onset. Figure $6 \mathrm{~A}$ shows PSTHs for an example MT cell at each frequency, plotted in linear time, with only slight changes in overall shape. Across all cells $(N=31)$, response latencies increased slightly (by $\sim 20 \mathrm{~ms})$ with frequency ( $p<0.001$; ANOVA) (Fig. 6B), while response durations decreased slightly $(p<0.05)$ (Fig. $6 C$ ). In contrast, when plotted with respect to sniff phase, PSTHs changed dramatically with frequency (Fig. 6D), as did response latencies and durations (Figs. 6E,F). Thus, latency-based odor representations are relatively invariant (and thus more robust) to sniff frequency, whereas phase-based representations are highly dependent on frequency.

\section{Increasing sniff frequency shorten responses of slowly activated MT cells}

The duration and rise time of the inhalation-driven MT response are likely critical for determining the ability of MT cells to follow sniffs at high frequency, as responses must reach a peak and return to baseline in the time between successive sniffs. Previously we observed a bimodal distribution of response durations for MT responses at $1 \mathrm{~Hz}$ sniffing (Fig. 3); we thus asked whether MT cells showing short-duration $(<100 \mathrm{~ms})$ and long-duration $(>100$ $\mathrm{ms}$ ) responses showed differential effects of sniff frequency on response patterns. The examples in Figure $7 A$ suggest that this is the case: the MT cell with a short-duration burst at $1 \mathrm{~Hz}$ shows relatively little change in PSTH shape as sniff frequency increases, while the cell with a longer-duration burst shows a marked change, with an overall shortening of response duration (Fig. $7 A$ ). Consistent with these examples, we found that MT cells with 
A

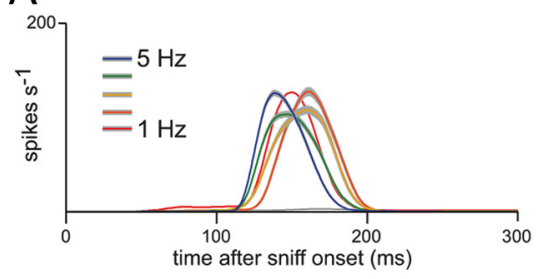

B
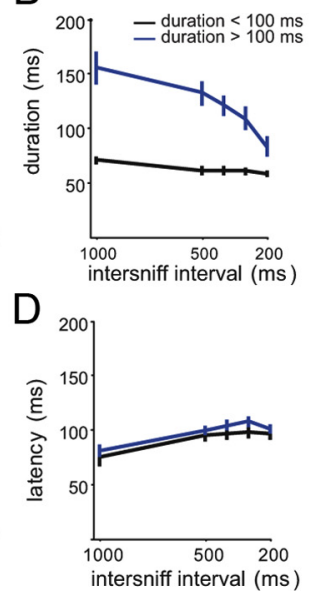

Figure 7. Sniff-evoked response duration separates MT cells with distinct effects of sniff frequency. $\boldsymbol{A}$, PSTHs at different frequencies for two MT cells with short (top) and long (bottom) response durations at $1 \mathrm{~Hz}$ sniffing. Mean $\pm \mathrm{SEM}$ of instantaneous firing rates are shown and color coded as in Figure 5; gray plots show preodor firing rate. For the short-duration cell, responses show only minor changes in shape with increasing sniff frequency. For the longer-duration cell, however, as sniff frequency is increased, the peak spike rate, response duration, and rise time all decrease, whereas latency increases. $\boldsymbol{B}, \boldsymbol{C}$, Effect of sniff frequency on MT cell response duration $(\boldsymbol{B})$ and rise times $(\boldsymbol{C})$, plotted separately for short-duration MT cells (duration $<100$ ms; black) and longer-duration cells (duration $>100 \mathrm{~ms}$; blue). Cells with shorter response durations (at $1 \mathrm{~Hz}$ ) show no change in duration or rise time with increased sniff frequency, whereas those with initially longer response durations show significantly decreased durations $(p<0.001$; ANOVA) and rise times ( $p<0.05$; paired $t$ test between 1 and $5 \mathrm{~Hz}$ ) at higher sniff frequencies. $\boldsymbol{D}, \boldsymbol{E}$, Effect of sniff frequency on response latency $(\boldsymbol{D})$ and onset precision $(\boldsymbol{E})$ for the same cells in $\boldsymbol{B}$ and $\boldsymbol{C}$. Shorter- and longer-duration cells have similar latencies and precision values. For shorter-duration cells, there is a small but significant increase in response latency $(\boldsymbol{D})$ with sniff frequency $(p<0.05$; ANOVA). Precision values $(\boldsymbol{E})$ are not affected by sniff frequency.

longer-duration responses at $1 \mathrm{~Hz}$ sniffing showed a significant decrease in response duration (Fig. $7 B)(p<0.001$; one-way ANOVA across frequency) and in response rise time (Fig. $7 C$ ) $(p<0.05$; ANOVA) at higher sniff frequencies. Mean response duration changed from $152 \mathrm{~ms}(1 \mathrm{~Hz})$ to $83 \mathrm{~ms}(5 \mathrm{~Hz})$, while mean rise time changed from $61 \mathrm{~ms}(1 \mathrm{~Hz})$ to $36 \mathrm{~ms}(5 \mathrm{~Hz})$. In contrast, MT cells with shorter-duration responses at $1 \mathrm{~Hz}$ showed no change in duration or rise time with frequency $(p>$ 0.2 for both; ANOVA). Consistent with this analysis, we also found a high concordance between those cells maintaining the highest modulation at $5 \mathrm{~Hz}$ and those showing short-duration responses at $1 \mathrm{~Hz}$ ( 17 of 19 short-duration cells showed low (SMI, $>0.90$ ) loss of modulation).

We also tested whether MT response latencies or onset precision changed with sniff frequency, separately analyzing MT cells with short- and longer-duration responses at $1 \mathrm{~Hz}$ (Figs. $7 \mathrm{D}, E$ ). There was a slight but significant increase in response latency with sniff frequency for both groups of MT cells $(80 \mathrm{~ms}$ for $1 \mathrm{~Hz}$ to $101 \mathrm{~ms}$ for $5 \mathrm{~Hz} ; p<0.05$, ANOVA) (Fig. 7D). We found no effect of sniff frequency on the precision of response onsets, however (Fig. 7E).

To confirm that the effects of sniff frequency generalize to natural sniffing patterns, in a subset of cells we presented odorant while performing sniff playback using the naturalistic sniff trace shown in Figure $1 A$. For analysis of frequency effects using this trace, we grouped the sniffs in the trace into one group containing all low-frequency sniffs (five sniffs per trace; mean sniff frequency, $1.6 \mathrm{~Hz}$ ) and the second containing high-frequency sniffs (14 sniffs per trace; mean, $5.8 \mathrm{~Hz}$ ). Between these two groups, peak firing rates decreased from a mean of $103 \pm 39 \mathrm{~Hz}$ during low-frequency sniffing to $72 \pm 28 \mathrm{~Hz}$ during high-frequency sniffing $(N=15$ cells). In addition, there was a clear separation of frequency effects as a function of MT response duration: response
C

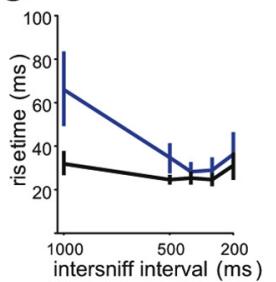

E

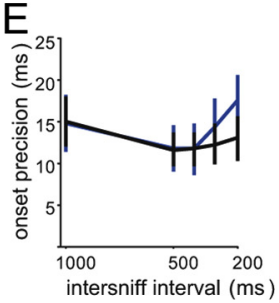

durations did not differ for the shorterduration MT cells (mean, $62 \pm 13 \mathrm{~ms}$ for low-frequency sniffing vs $67 \pm 18 \mathrm{~ms}$ for high-frequency sniffing; $N=11$ cells), but decreased for longer-duration MT cells (mean, $137 \pm 17 \mathrm{~ms}$ during lowfrequency sniffing vs $80 \pm 31 \mathrm{~ms}$ for highfrequency sniffing; $N=4$ cells).

In summary, we found using both the synthetic and naturalistic sniff playback traces that higher sniff frequencies lead to a reduction in the duration of the inhalation-evoked spike burst as well as a reduction in the rise time of the burst. Together, these effects lead to a shortening of the inhalation-driven burst, and they occur only for those MT cells that show longer-duration responses at low sniff frequency.

\section{Dynamics of sniff-driven response patterns across the MT cell population} Given the diversity in response dynamics and the change in these dynamics with sniff frequency, we asked how these responses would appear across a population of MT cells. To visualize this, we generated population spike histograms from all units, independent of odorant, using either the sniff bout trace or the "natural" sniffing trace (see Materials and Methods), with the rationale that this histogram would approximate the coherent temporal pattern of activity across a MT cell population during odorant sampling (Fig. $8 \mathrm{~A}$ ). Despite the fact that different MT cells have a range of excitation onset latencies, rise times, and durations, the population activity pattern followed sniffs with high reliability (Fig. $8 \mathrm{~A}$ ), showing sharp peaks in activity following each inhalation even during $5 \mathrm{~Hz}$ sniffing and during the high-frequency sniff bouts expressed in the awake rat (Fig. 8C).

Do the changes in PSTH shape contribute to the temporal patterning of MT cells as sniff frequency increases? To test this, we constructed synthetic response histograms using, for each unit, the mean PSTH evoked during $1 \mathrm{~Hz}$ sniffing and convolving this response (with a small activity-dependent attenuation factor) with the sniff times in the higher-frequency bouts (see Materials and Methods). At high sniff frequencies, the synthetic responses showed significantly less modulation by each sniff than the actual responses (mean SMI at $5 \mathrm{~Hz}$ sniffing, $0.80 \pm 0.18$ simulated responses vs $0.93 \pm 0.10$ actual responses; $p<0.001$, paired $t$ test; $N=37$ cells). The synthetic population histogram also showed less modulation than the actual population histogram (Figs. $8 B, C$ ). Thus, the frequency-dependent change in shape of the inhalation-evoked PSTH has the effect of increasing the ability of a population of MT cells to maintain coherent temporal patterning in spike timing as sniff frequency increases.

Because awake, behaving rats sample odorants at frequencies up to $10 \mathrm{~Hz}$ (Kepecs et al., 2007; Wesson et al., 2008b, 2009), we used the same approach to extrapolate population-level response dynamics at frequencies higher than the range that we could test experimentally using sniff playback. We extended the synthetic response histograms to $10 \mathrm{~Hz}$ sniffing (convolving each PSTH with sniff times repeated at $10 \mathrm{~Hz}$ ) using either 1 or $5 \mathrm{~Hz}$ PSTHs from the same cells (Fig. 8D). For each, we measured the SMI at 

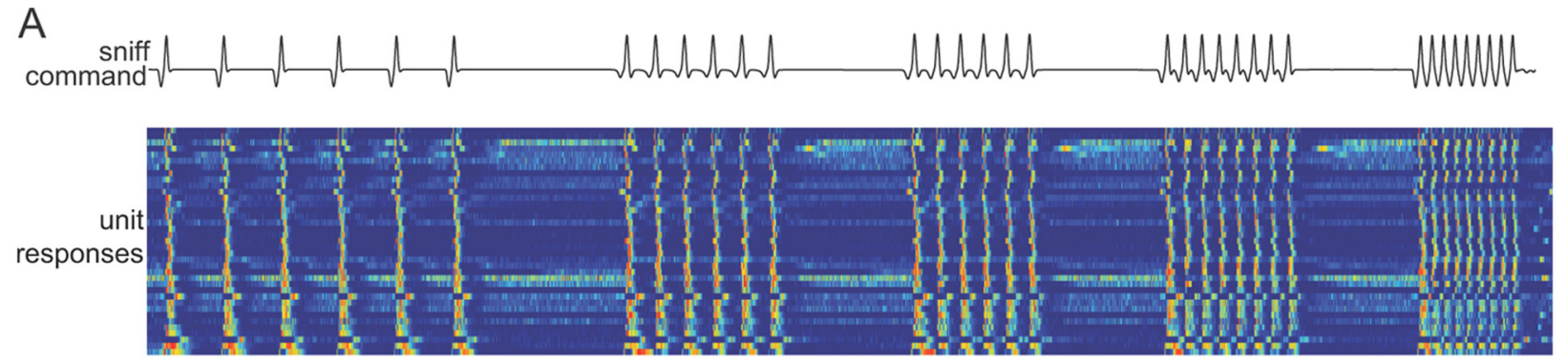

population response

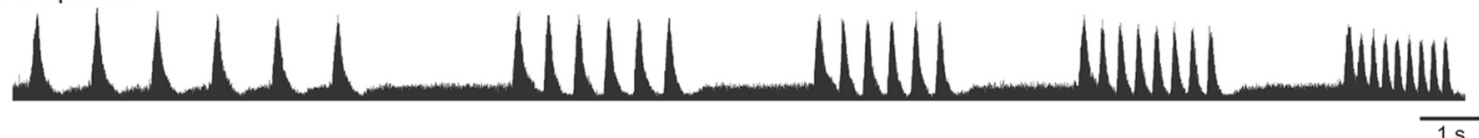

B

synthetic population response

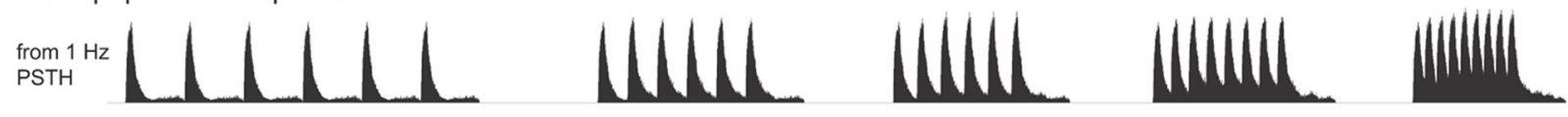

C

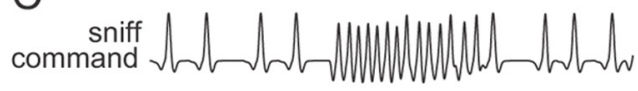

population response

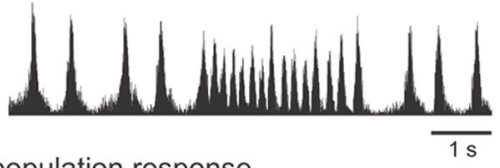

synthetic population response

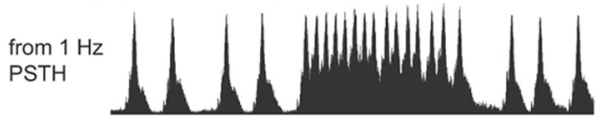

$\mathrm{E}$

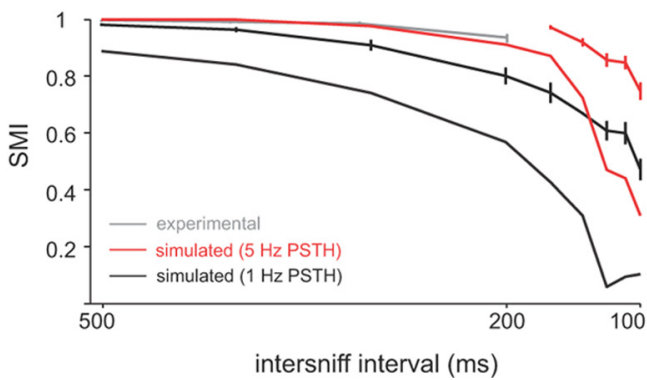

extrapolated population activity
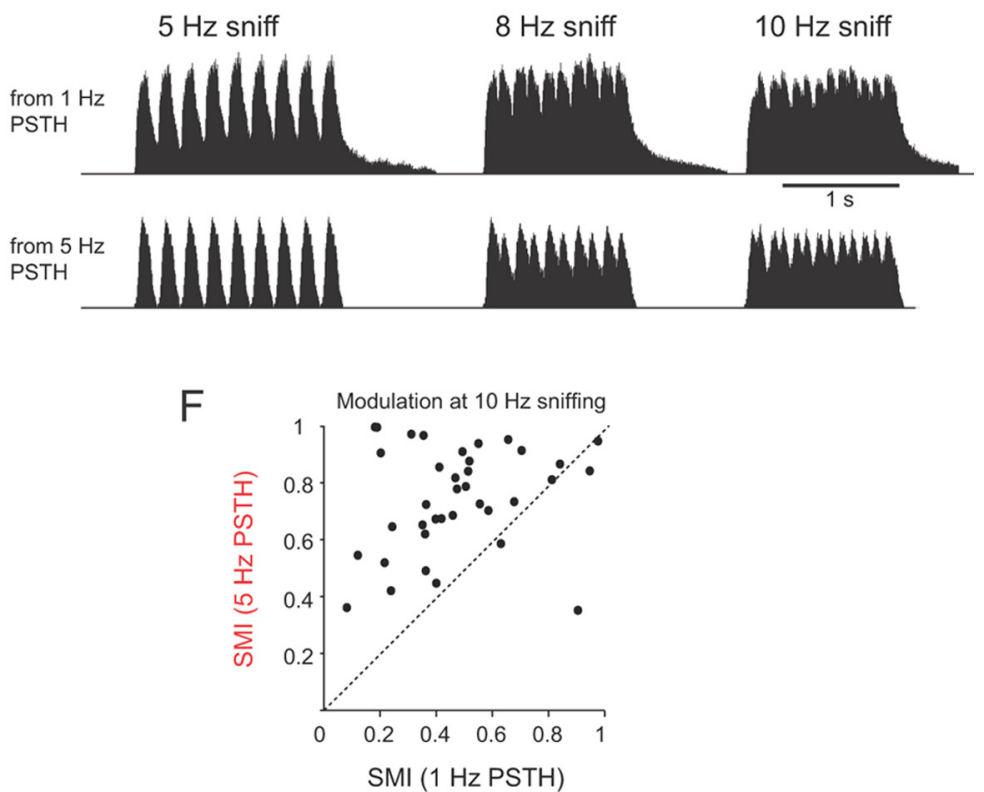

Figure 8. Frequency-dependent transformations in response dynamics enable robust temporal patterning across the MT cell population. $A$, Activity evoked by the same sniff playback trace across all recorded units $(N=37$ cells), compiled independently of odorant identity or concentration. Top, Playback command trace. Middle, Pseudocolor representation of firing rates ( $5 \mathrm{~ms}$ bin) for each unit, normalized to peak firing rate for each unit, one unit per row. Units are ordered by single-sniff response duration, from shortest (top) to longest. Warm colors indicate peak firing rates. Bottom, Population activity histograms generated by averaging the response histograms from all units. Each histogram was normalized to its peak firing rate before averaging. $\boldsymbol{B}$, "Synthetic" response histogram constructed by convolving the $1 \mathrm{~Hz}$ PSTHs with the sniff times for each unit and then averaging these activity patterns across all units. This approach predicts population-level response patterns based solely on the responses at $1 \mathrm{~Hz}$ sniffing. Comparing the synthetic patterns with the actual recorded responses (as in $A$ ) indicates the degree to which frequency-dependent changes in responses shape population-level activity. Responses constructed from $1 \mathrm{~Hz}$ PSTHs show less modulation with sniffing compared to actual responses (compare to $A$ ). $C$, Population activity histograms during playback of the natural sniffing sequence. Top, Playback command trace. Middle, Population activity histogram constructed as for $\boldsymbol{A}$. Bottom, Synthetic response histogram constructed from $1 \mathrm{~Hz}$ PSTHs as for $\boldsymbol{B}$. Again, activity across the MT population maintains strong patterning and modulation with sniffing, whereas the synthetic population pattern shows less modulation. $\boldsymbol{D}$, Extrapolation of population responses to sniff frequencies up to $8 \mathrm{~Hz}$ using the PSTH convolution method. Synthetic population response histograms were constructed as in $\boldsymbol{A}$ using either $1 \mathrm{~Hz}$ (top) or $5 \mathrm{~Hz}$ (bottom) PSTHs from the same units by convolving with sniff times repeated at 8 and $10 \mathrm{~Hz}$. Population responses show weak modulation with sniffing using the $1 \mathrm{~Hz}$ PSTHs $(S M I$ at $8 \mathrm{~Hz}, 0.16$ ) but stronger modulation using the $5 \mathrm{~Hz}$ PSTHs (SMI, 0.64). E, SMl values for all units as a function of frequency measured experimentally (gray), simulated using $1 \mathrm{~Hz}$ PSTHs (black) and $5 \mathrm{~Hz}$ PSTHs (red). SMI values were measured for frequencies extrapolated up to $10 \mathrm{~Hz}$ by convolution (see Materials and Methods). Traces with error bars show SMI values measured across individual units; those without error bars show the SMI value for the population-averaged response. SMI values were higher for the experimental data and for responses synthesized from $5 \mathrm{~Hz}$ PSTHS. F, SMI values for $10 \mathrm{~Hz}$ sniffing extrapolated for each unit using either 1 or $5 \mathrm{~Hz}$ PSTHs. For nearly all units, the SMI value was higher using the $5 \mathrm{~Hz}$ PSTH.

frequencies up to $10 \mathrm{~Hz}$ (Fig. 8 E). Population-level response patterning was almost entirely lost at $10 \mathrm{~Hz}$ if the $1 \mathrm{~Hz}$ PSTH responses were used, but patterning remained strong using the $5 \mathrm{~Hz}$ PSTH responses. These effects were clear for individual MT cells as well: nearly all cells showed greater modulation for $10 \mathrm{~Hz}$ sniffs when the synthetic responses were based on the $5 \mathrm{~Hz}$ PSTH than when based on the $1 \mathrm{~Hz}$ PSTH (Fig. $8 F$ ). These analyses indicate that MT response patterns evoked by sniffing at $5 \mathrm{~Hz}$ are better able to follow sniffs at these and higher frequencies than are response patterns evoked by sniffing at $1 \mathrm{~Hz}$. 


\section{Discussion}

In previous studies characterizing the temporal structure of sensory input to the $\mathrm{OB}$, we found that ORN inputs are tightly coupled to inhalation, have intrinsic differences in their dynamics, and are qualitatively altered by changes in sniff frequency (Spors et al., 2006; Verhagen et al., 2007; Carey et al., 2009). These findings led to predictions about the relationship between sensory input dynamics and postsynaptic activity, and about how sniff frequency might affect MT responses. Here we tested those predictions by recording MT responses while manipulating sniffing in anesthetized rats, using sniff waveforms taken from awake rats. Several of our observations support earlier predictions: we found that inhalation-driven MT responses, like ORN inputs, show intrinsic differences in their response latency, rise time, and duration that vary over a similar range, and that high-frequency sniffing attenuates the magnitude of the MT response. Other predictions - in particular, that temporal patterning of MT responses would diminish at high frequencies-were not supported. Instead, most MT cells maintained strong temporal patterning of responses at all frequencies tested; this was accompanied by changes in the shape of sniff-evoked responses as sniff frequency increased.

High-frequency sniffing is a hallmark feature of active odor sampling, yet only a few studies have attempted to characterize how changes in sniff frequency affect olfactory processing. Recordings from awake, freely moving rats have yielded mixed results on the effect of sniff frequency, with some reports that temporal patterning of $\mathrm{OB}$ neurons is diminished at high frequencies (Bhalla and Bower, 1997; Kay and Laurent, 1999), and one other report that patterning persists (Cury and Uchida, 2010). Resolving these differences is difficult because frequency effects were not analyzed systematically and because of the potential confound of behavioral state-dependent modulation of responses (Bhalla and Bower, 1997; Kay and Laurent, 1999). A previous study using artificial sniffing in anesthetized rats found that most MT cells maintain respiratory patterning at moderate $(3 \mathrm{~Hz})$ and high $(6 \mathrm{~Hz})$ sniff frequencies, with a reduction in response magnitude (Bathellier et al., 2008).

Our results extend these earlier reports by establishing that inhalation-driven responses change significantly with sniff frequency and that these changes are driven by a "bottom-up" pathway related to active sampling, rather than "top-down" neuromodulatory pathways, which can also shape MT spike timing in awake animals (Doucette et al., 2011). For many cells - in particular, those that maintained the strongest temporal patterning at high sniff frequencies - the duration and the rise time of the inhalation-driven response shortened, leading to a temporal sharpening of activity. Thus, odorant-evoked response patterns of MT cells change with sampling behavior. This conclusion contrasts with a recent characterization of MT responses in behaving rats, which reported that firing patterns were frequency invariant (Cury and Uchida, 2010). That study, however, focused only on the first in a bout of high-frequency sniffs. Here, we found that the first sniff in any bout elicits equivalent responses regardless of the frequency of subsequent sniffs; we reported the same result previously for ORN inputs (Verhagen et al., 2007). Responses to later sniffs, however, do depend on recent sniff history.

One parameter of MT response dynamics that remained invariant to sniff frequency was onset latency relative to inhalation. This invariance is consistent with MT response timing depending on ORN inputs, which also show frequency-invariant latencies (Carey et al., 2009). The fixed timing of MT responses relative to inhalation constrains models of temporal coding that rely on the phase of MT activity relative to the respiratory cycle (Chaput, 1986; Hopfield, 1995). These models have demonstrated robust phase-based coding of odor information by MT response onsets but have typically considered only cases of regular respiration rates. In behaving rodents, sniffs are rarely repeated at regular intervals, and sniff frequency can vary dramatically from one sniff to the next (Welker, 1964; Kepecs et al., 2007; Wesson et al., 2008b). One proposal for adapting phase-based coding schemes to the diverse and irregular sniffing patterns has been to translate absolute timing into phase-based timing using the period between two successive inhalations (Roux et al., 2006; Fantana et al., 2008). We found that translating MT responses onto a phase time base eliminated the frequency invariance in response latencies as well as response durations. These results, along with others arising from awake, behaving animals, suggest that information coding by MT responses appears to be organized in terms of absolute timing relative to inhalation rather than relative to a respiratory "cycle" (Margrie and Schaefer, 2003; Cury and Uchida, 2010; Junek et al. 2010).

At the population level, the net effect of frequency-dependent changes in MT responses was to enhance respiratory patterning. Responses evoked by $1 \mathrm{~Hz}$ sniffing were significantly less modulated when repeated at higher frequency than responses during 5 $\mathrm{Hz}$ sniffing. Furthermore, $5 \mathrm{~Hz}$ sniff-evoked responses were able to maintain significant patterning even when repeated at $10 \mathrm{~Hz}$ (Fig. 8). Thus, the frequency-dependent temporal sharpening of MT spike bursts enables coherent population-level spiking dynamics to remain linked to each inhalation across the range of sniff frequencies expressed during behavior.

What changes in the inhalation-evoked MT response underlie their ability to maintain respiratory patterning across frequencies? The two parameters that changed most consistently were duration and rise time: MT responses reached a peak more quickly and shut down more quickly at high sniff frequencies. MT response rise times and durations were strongly correlated, with faster rise times correlating with shorter durations. Several pieces of evidence suggest that these frequency-dependent changes are mediated by $\mathrm{OB}$ postsynaptic circuitry rather than frequency-dependent changes in sniff waveform or presynaptic activity patterns. First, MT responses to the first sniff in a bout did not change shape with frequency, despite the fact that sniff waveform did change slightly. Second, ORN inputs did not show substantial changes in shape as frequency increased (Fig. 1G), and presynaptic inhibition of ORN inputs does not change with sniff frequency (Pírez and Wachowiak, 2008). Third, frequencydependent effects on duration and rise time were apparent only for a subset of MT cells, in particular, cells with relatively long ( $>100 \mathrm{~ms})$ response durations at low sniff frequencies.

One candidate circuit mediating these effects involves connections between external tufted (ET) cells, inhibitory periglomerular interneurons, and MT cells. ET cells are excitatory interneurons receiving strong ORN input and directly exciting periglomerular interneurons within the same glomerulus (Hayar et al., 2004b). ET cells show entrainment of spike bursts to rhythmic ORN inputs (Hayar et al., 2004a). Thus, an increase in ET cell burst frequency as sniff frequency increases is predicted to cause stronger and more synchronous activation of periglomerular interneurons, leading in turn to a shortened MT cell response as sniff frequency increases and enhanced respiratory modulation at higher sniff frequencies (Wachowiak and Shipley, 2006). The results observed here support these predictions. Alternatively, temporal sharpening might be mediated by recurrent inhibition between MT and granule cells. Recurrent inhibition can enhance the temporal precision of MT cell firing in the face of rhythmic ORN inputs (Balu et al., 2004; Schoppa, 2006; David et al., 2008), and there is evidence that recurrent inhibition is stronger at 
higher sniff frequencies (Young and Wilson, 1999). In either case, ORN inputs that are more synchronous would lead to faster activation of MT cells and also to stronger recruitment of the feedforward or recurrent inhibitory circuits that shorten the MT cell response, consistent with the correlation we measured between response rise time, duration, and the degree of frequency-dependent temporal patterning (Figs. 3, 5). One set of predictions that follows from this model is that the rise time of ORN inputs to a glomerulus should correlate with that of MT cells innervating the same glomerulus, and glomeruli receiving faster ORN inputs should show stronger modulation of MT cell responses at high sniff frequencies. These predictions need to be tested by comparing ORN inputs to and MT cell responses for the same glomerulus.

Odorant sampling in behaving animals is a dynamic but precisely controlled behavior that presumably optimizes the detection and processing of sensory information. How might frequency-dependent attenuation and temporal sharpening of MT spike bursts optimize odor information processing? First, maintaining (and even enhancing) the coherence of MT spiking with sniffing has been shown to enhance the precision of MT spike timing, thus increasing the potential discriminatory power of MT spike trains (Hopfield, 1995; Schaefer et al., 2006). Second, temporally patterned MT spiking appears to be important for information processing downstream of MT cells, for example, the temporal integration of inputs from multiple MT cells by pyramidal neurons in piriform cortex (Luna and Schoppa, 2008; Stokes and Isaacson, 2010). Third, frequency-dependent changes in MT response patterns may leave odor identity coding unchanged but enhance the ability of the population to process other aspects of odor information relevant to active sensing. For example, the reduction in MT response magnitude may reduce the salience of odorants that are constant across a highfrequency sniff bout while maintaining sensitivity to newly encountered odorants (Verhagen et al., 2007). Finally, high-frequency, temporally patterned MT bursts may be important in driving synaptic plasticity within the $\mathrm{OB}$ or its targets during odor learning. Further electrophysiological experiments combined with behavioral analyses will be important to test these possibilities.

\section{References}

Balu R, Larimer P, Strowbridge BW (2004) Phasic stimuli evoke precisely timed spikes in intermittently discharging mitral cells. J Neurophysiol 92:743-753.

Bathellier B, Buhl DL, Accolla R, Carleton A (2008) Dynamic ensemble odor coding in the mammalian olfactory bulb: sensory information at different timescales. Neuron 57:586-598.

Bhalla US, Bower JM (1997) Multiday recordings from olfactory bulb neurons in awake freely moving rats: spatially and temporally organized variability in odorant response properties. J Comput Neurosci 4:221-256.

Carey RM, Verhagen JV, Wesson DW, Pirez N, Wachowiak M (2009) Temporal structure of receptor neuron input to the olfactory bulb imaged in behaving rats. J Neurophysiol 101:1073-1088.

Chaput MA (1986) Respiratory-phase-related coding of olfactory information in the olfactory bulb of awake freely-breathing rabbits. Physiol Behav 36:319-324.

Cheung MC, Carey RM, Wachowiak M (2009) A method for generating natural and user-defined sniffing patterns in anesthetized or reduced preparations. Chem Senses 34:63-76.

Cury KM, Uchida N (2010) Robust odor coding via inhalation-coupled transient activity in the mammalian olfactory bulb. Neuron 68:570-585.

David F, Linster C, Cleland TA (2008) Lateral dendritic shunt inhibition can regularize mitral cell spike patterning. J Comput Neurosci 25:25-38.

Davison IG, Katz LC (2007) Sparse and selective odor coding by mitral/ tufted neurons in the main olfactory bulb. J Neurosci 27:2091-2101.

Doucette W, Gire David H, Whitesell J, Carmean V, Lucero Mary T, Restrepo D (2011) Associative cortex features in the first olfactory brain relay station. Neuron 69:1176-1187.

Fantana AL, Soucy ER, Meister M (2008) Rat olfactory bulb mitral cells receive sparse glomerular inputs. Neuron 59:802-814.
Hayar A, Karnup S, Shipley MT, Ennis M (2004a) Olfactory bulb glomeruli: external tufted cells intrinsically burst at theta frequency and are entrained by patterned olfactory input. J Neurosci 24:1190-1199.

Hayar A, Karnup S, Ennis M, Shipley MT (2004b) External tufted cells: A major excitatory element that coordinates glomerular activity. J Neurosci 24:6676-6685.

Hopfield JJ (1995) Pattern recognition computation using action potential timing for stimulus representation. Nature 376:33-36.

Junek S, Kludt E, Wolf F, Schild D (2010) Olfactory coding with patterns of response latencies. Neuron 67:872-884.

Kay LM, Laurent G (1999) Odor- and context-dependent modulation of mitral cell activity in behaving rats. Nat Neurosci 2:1003-1009.

Kepecs A, Uchida N, Mainen ZF (2007) Rapid and precise control of sniffing during olfactory discrimination in rats. J Neurophysiol 98:205-213.

Luna VM, Schoppa NE (2008) GABAergic circuits control input-spike coupling in the piriform cortex. J Neurosci 28:8851-8859.

Macrides F, Eichenbaum HB, Forbes WB (1982) Temporal relationship between sniffing and the limbic theta rhythm during odor discrimination reversal learning. J Neurosci 2:1705-1711.

Margrie TW, Schaefer AT (2003) Theta oscillation coupled spike latencies yield computational vigour in a mammalian sensory system. J Physiol 546:363-374.

Pírez N, Wachowiak M (2008) In vivo modulation of sensory input to the olfactory bulb by tonic and activity-dependent presynaptic inhibition of receptor neurons. J Neurosci 28:6360-6371.

Roux SG, Garcia S, Bertrand B, Cenier T, Vigouroux M, Buonviso N, Litaudon P (2006) Respiratory cycle as time basis: an improved method for averaging olfactory neural events. J Neurosci Methods 152:173-178.

Schaefer AT, Angelo K, Spors H, Margrie TW (2006) Neuronal oscillations enhance stimulus discrimination by ensuring action potential precision. PLoS Biology 4:e163.

Schoppa NE (2006) Synchronization of olfactory bulb mitral cells by precisely timed inhibitory inputs. Neuron 49:271-283.

Schoppa NE, Westbrook GL (2001) Glomerulus-specific synchronization of mitral cells in the olfactory bulb. Neuron 31:639-651.

Sobel EC, Tank DW (1993) Timing of odor stimulation does not alter patterning of olfactory bulb unit activity in freely breathing rats. J Neurophysiol 69:1331-1337.

Spors H, Wachowiak M, Cohen LB, Friedrich RW (2006) Temporal dynamics and latency patterns of receptor neuron input to the olfactory bulb. J Neurosci 26:1247-1259.

Stokes CCA, Isaacson JS (2010) From dendrite to soma: dynamic routing of inhibition by complementary interneuron microcircuits in olfactory cortex. Neuron 67:452-465.

Verhagen JV, Wesson DW, Netoff TI, White JA, Wachowiak M (2007) Sniffing controls an adaptive filter of sensory input to the olfactory bulb. Nat Neurosci 10:631-639.

Wachowiak M, Cohen LB (2001) Representation of odorants by receptor neuron input to the mouse olfactory bulb. Neuron 32:723-735.

Wachowiak M, Shipley MT (2006) Coding and synaptic processing of sensory information in the glomerular layer of the olfactory bulb. Semin Cell Dev Biol 17:411-423.

Walker JK, Lawson BL, Jennings DB (1997) Breath timing, volume and drive to breathe in conscious rats: comparative aspects. Respir Physiol 107:241-250.

Welker WI (1964) Analysis of sniffing in the albino rat. Behavior 22:223-244.

Wesson DW, Carey RM, Verhagen JV, Wachowiak M (2008a) Rapid encoding and perception of novel odors in the rat. PLoS Biology 6:e82.

Wesson DW, Donahou TN, Johnson MO, Wachowiak M (2008b) Sniffing behavior of mice during performance in odor-guided tasks. Chemical Senses 33:581-596.

Wesson DW, Verhagen JV, Wachowiak M (2009) Why sniff fast? The relationship between sniff frequency, odor discrimination, and receptor neuron activation in the rat. J Neurophysiol 101:1089-1102.

Yaksi E, Friedrich RW (2006) Reconstruction of firing rate changes across neuronal populations by temporally deconvolved $\mathrm{Ca}^{2+}$ imaging. Nat Methods 3:377-383.

Young TA, Wilson DA (1999) Frequency-dependent modulation of inhibition in the rat olfactory bulb. Neurosci Lett 276:65-67.

Youngentob SL, Mozell MM, Sheehe PR, Hornung DE (1987) A quantitative analysis of sniffing strategies in rats performing odor discrimination tasks. Physiol Behav 41:59-69. 